\title{
Androginia psicológica y flexibilidad comportamental
}

\section{Julia Sebastián \\ Concha Aguiñiga \\ Bernardo Moreno}

\section{ANDROGINIA PSICOLOGICA Y FLEXIBILIDAD COMPORTAMENTAE}

La nueva formulación de los roles sexuales, más concretamente, de la masculinidad y feminidad, surgida en la década de los setenta, se basa en una serie de presupuestos (bidimensionalidad, ortogonalidad, independencia con respecto al sexo biológico, etc.) que han hecho posible la conceptualización del rol sexual andrógino.

La suposición tradicional de que la masculinidad y la feminidad representaban extremos opuestos de una única dimensión fue criticada y contestada con una nueva proposición sobre la independencia de ambos constructos (Constantinople, 1973). La estrecha relación que el modelo clásico defendía entre sexo del sujeto, género (masculinidad-feminidad), y salud mental, proponiendo que la feminidad para la mujer y la masculinidad para el hombre representaban la salud o bienestar psicológico individual, fue trastocada por los resultados empíricos que apuntaban la mayor ventaja psicosocial de los sujetos andróginos y/o masculinos independientemente de su sexo.

Por otra parte, la práctica común de interpretar la masculinidad como lo opuesto a la feminidad y viceversa - ya que se suponían estrechamente vinculadas con la realidad biológica - tuvo que ser abandonada: la masculinidad no es lo contrario de la feminidad, ni la feminidad es la no masculinidad.

La existencia de ambos constructos por separado (bidimensionalidad, y la independencia entre ellos (ortogonalidad), además de favorecer un mayor alejamiento del sexo biológico y de sus implicaciones, posibilitó una cuádruple clasificación del sujeto: masculino, femenino, indiferenciado y andrógino.

Numerosos instrumentos han sido elaborados a partir de estos presupuestos implícitos de los constructos de masculinidad y feminidad: el Inventario de Rol Sexual (BSRI; Bem, 1974), el Cuestionario de Atributos Personales (PAQ; Spence, Helmreich y Stapp, 1974), la Escala de Comportamiento del Rol Sexual (SRBS; Orlofsky, 1981), la Escala PRF ANDRO (Berzins, Welling y Wetter, 1978), las Escalas de Masculinidad (MSC) y Feminidad (FMN) del CPI (Baucom, 1976), y las Escalas de Masculinidad y Feminidad del Adjective Check List (ACL; Heilbrun, 1976).

Todos ellos se han basado en mayor o menor medida en la distinción entre roles expresivos e instrumentales propuesto por Parson y Bales (1955) como los dominios característicos de hombres y mujeres, o en lo que otros autores han denominado «agency» - lo que concierne a uno mismo y a la consecución de las propias metas-y «communal» - lo que concierne a uno pero en relación con los otros- (Bakan, 1966). En este mismo sentido, Erikson 
(1964) habló de espacio externo y espacio interno, y Koestler (1967) de tendencias autoasertivas y tendencias integrativas.

Precisamente una nota diferencial con respecto al modelo clásico de la masculinidad y feminidad es que estos constructos son ahora definidos socioculturalmente, y no por medio de un criterio estadístico (diferencias estadísticamente significativas entre hombres y mujeres en las respuestas) como sucedía anteriormente. Los items de las nuevas escalas representan características socialmente deseables para varones $\mathrm{y} / \mathrm{o}$ mujeres.

El mayor logro y reconocimiento que ha supuesto el nuevo planteamiento sobre los roles sexuales ha sido quizá el concepto de androginia. El rol sexual andrógino o, lo que es lo mismo, la integración de atributos definidos culturalmente como masculinos y femeninos, constituyó el centro de atención de los investigadores interesados en este tema, desarrollándose una gran cantidad de trabajos con el objeto de validar conceptualmente, y en menor medida comportamentalmente, el constructo.

Su operativización fue llevada a cabo en primer lugar por Bem, mediante la utilización de su cuestionario BSRI (Bem, 1974). Paralelamente a su intento, otro grupo de investigadores desarrollaron una labor muy semejante dando lugar al segundo cuestionario en importancia en la evaluación de la orientación del rol sexual del sujeto: el PAQ (Spence, Helmreich y Stapp, 1974, 1975). Fueron precisamente estos últimos autores quienes propusieron la metodología - comúnmente utilizada hoy en día, aunque no sin bastantes detractores - de utilizar la mediana como índice a partir del cual categorizar al sujeto como masculino (aquellos individuos que puntúan por encima de la mediana en la escala de Masculinidad y por debajo de la mediana en la escala de Feminidad), femeninos (aquellos que puntúan por encima en la escala de Feminidad y por debajo en la de Masculinidad), andróginos (los que puntúan por encima de la mediana tanto en la escala de Masculinidad como en la de Feminidad) e indiferenciados (los que puntúan por debajo en ambas escalas).

El concepto de androginia estuvo asociado casi desde sus comienzos con un mayor bienestar psicológico. Fue precisamente Sandra Bem la primera que planteó la necesidad de revisar el concepto de salud mental para tratar de poner de manifiesto la equiparación que de hecho existe entre ésta y los rasgos «amparados» bajo el concepto de masculinidad (Bem, 1975). Su propuesta sobre la persona andrógina como el prototipo humano de salud mental fue muy bien acogida, como lo demuestra la cantidad ingente de publicaciones que aparecieron con el objeto de verificar tal posibilidad.

Las causas de la «explosión bibliográfica» que aconteció a finales de la década de los setenta (fundamentalmente en el año de 1979) se debió no sólo a la posibilidad de cuantificar el rol sexual andrógino - hasta esos momentos únicamente analizado a través de religiones, filosofías, leyendas o mitossino también a la asociación empírica que empezaba a mostrarse entre éste $\mathrm{y}$ un mayor ajuste psicosocial.

La androginia fue proclamada con entusiasmo como una panacea en el campo de la salud mental, ya que comportaba una mayor eficacia personal y social. La madurez y el equilibrio personal iban a depender, desde esta perspectiva, de la armonía de características masculinas y femeninas, tanto para el hombre como para la mujer. Sin embargo, muy pronto hubo que descender al campo de los datos empíricos y reconocer que era el rol sexual masculino - y no tanto el andrógino- el que resultaba favorecido en los estudios realizados.

El vínculo existente entre androginia y salud mental se producía a través de la llamada «flexibilidad o adaptabilidad comportamental»; característica 
sólo asumible por el rol sexual andrógino debido a que su autoconcepto no restringía los comportamientos apropiados o no socialmente a su sexo.

Bem postuló que los individuos con una tipificación sexual muy marcada se encuentran limitados en cuanto al rango de comportamientos que se permiten llevar a cabo (Bem, 1975, 1976), debido a un proceso motivacional que les impele a guardar un comportamiento consistente con un estándar del rol sexual internalizado (Kagan, 1964; Kohlberg, 1966). Esta restricción comportamental es llevada a cabo mediante la represión de cualquier comportamiento que pueda ser considerado como no deseable o inapropiado para su sexo.

Un autoconcepto de masculinidad o de feminidad restringido inhibirá aquellos comportamientos que han sido considerados como típicos o pertenecientes a uno de los dos sexos (estereotipo), mientras que un autoconcepto mixto o andrógino permitirá al sujeto actuar con cierta libertad y desarrollar cualquier tipo de comportamiento.

Los individuos varian en el grado en el que utilizan las definiciones culturales de masculinidad y feminidad para evaluar su propia personalidad y comportamiento. Los individuos tipificados sexualmente están en gran armonía con estas definiciones y procuran que sus comportamientos sean consistentes con ellas, mediante la selección de conductas y atributos que refuercen esta imagen y por la evitación de todo aquello que la viole. En contraste, los individuos andróginos están menos armonizados con estas definiciones culturales de feminidad y masculinidad y es menos probable que regulen su comportamiento de acuerdo a ellas. Son capaces de realizar cualquier tipo de comportamiento efectivo para una situación, sin tener en cuenta el estereotipo como apropiado para un sexo o para otro.

En este sentido, la atribución de un ítem del BSRI es interpretado por Bem como indicativo de hasta qué punto los estereotipos sexuales han sido internalizados y consecuentemente controla el comportamiento prescribiendo y proscribiendo varios actos.

Así, pues, los trabajos iniciales de Bem tuvieron como objetivo verificar la supuesta flexibilidad comportamental de los sujetos andróginos. Y esto fue realizado en dos momentos diferentes. Primero, estableció la adaptabilidad de los individuos andróginos tanto en tareas masculinas como femeninas (Bem, 1975b; Bem, Martyna y Watson, 1976). Posteriormente intentó comprobar la evitación y el «disconfort» que sufren los sujetos tipificados al enfrentarse con tareas no apropiadas a su sexo (Bem y Lenney, 1976).

El primer experimento fue diseñado para evocar un comportamiento estereotípicamente masculino: la independencia a las presiones sociales, y un comportamiento estereotípicamente femenino: la interacción con un gatito (Bem, 1975c).

Las hipótesis fueron, para el primer caso, que los sujetos masculinos y andróginos permanecerían más independientes a una presión social (una situación de conformidad social basada en la clasificación de series humorísticas) que los sujetos femeninos y, para el segundo, que los sujetos femeninos y andróginos serían más cariñosos y juguetones con el gatito que los sujetos masculinos. En definitiva, lo que se intentaba comprobar era que sólo los sujetos andróginos realizarían ambos tipos de pruebas de una manera adecuada sin tener en cuenta el estereotipo del rol sexual en los comportamientos particulares. Por el contrario, los sujetos tipificados sexualmente como masculinos o femeninos sólo realizarían bien aquella situación que exige un comportamiento que es congruente con su autodefinición como masculino y femenino.

Dos comparaciones ortogonales fueron realizadas y confirmadas a partir de este experimento. Primero, los sujetos masculinos y andróginos estuvieron de acuerdo con la opinión de los demás sujetos significativamente en me- 
nos ensayos que los sujetos femeninos y, segundo, los sujetos masculinos y andróginos no difirieron entre sí en su grado de conformidad. Estas comparaciones fueron significativas para los varones, para las mujeres y para los sexos combinados.

En la segunda situación las cosas fueron algo más complicadas. Pese a que las comparaciones ortogonales realizadas fueron que los sujetos femeninos y andróginos mostrarían una mayor involucración con el gatito que los sujetos masculinos y, que los sujetos femeninos y andróginos no diferirían entre sí, los resultados fueron algo diferentes según el sexo de los sujetos, y en menor medida según su género.

Entre los varones, los sujetos femeninos y andróginos mostraron significativamente una mayor implicación total con el gatito que los varones masculinos, tal y como se predijo, no existiendo diferencias significativas entre varones femeninos y andróginos. Por lo tanto, las dos hipótesis quedaron comprobadas.

En cuanto a las mujeres, no se verificaron ninguna de las dos hipótesis previstas, ya que las mujeres femeninas mostraron significativamente menos involucración total con el gatito que las mujeres andróginas. Los resultados para las mujeres masculinas fueron muy ambiguos.

Sin embargo, la hipótesis central de Bem de que los sujetos andróginos serían más flexibles comportamentalmente a través de varias situaciones que los sujetos tipificados, parece confirmarse, ya que sólo estos sujetos de ambos sexos fueron al mismo tiempo independientes en su criterio y afectivos-lúdicos con el gatito. Para los sujetos no andróginos las hipótesis no fueron totalmente confirmadas. En el caso de los varones no hubo problemas, pero en el caso de las mujeres y en lo que respecta únicamente a la tarea femenina sí aparecieron algunas discrepancias.

Los datos del experimento anterior llevaron a Bem y colaboradores (Bem, Martyna y Watson, 1976) a realizar otro experimento de similares características para poder explicar algunos de sus resultados. En esta ocasión, se daba la oportunidad a la mujer femenina a que desarrollase un comportamiento expresivo variando sustancialmente el contexto. La primera situación se refería a la interacción del sujeto con un bebé, mientras que la segunda requería escuchar a un estudiante que contaba algunas de sus desdichas.

En cuanto a la primera situación, los resultados no verificaron ninguna de las hipótesis propuestas: los sujetos femeninos y andróginos de ambos sexos no fueron más afectivos con el bebé que los sujetos masculinos, y entre los dos grupos primeros aparecieron diferencias en cuanto a esta cuestión.

Posteriormente Bem procedió a distinguir a los sujetos andróginos de los indiferenciados (aquellos que puntúan por encima de la mediana en $\mathrm{M}$ y $\mathrm{F}$, y aquellos que lo hacen por debajo), siguiendo las directrices de Spence y colaboradores (1975). Al reanalizar los datos desde esta nueva perspectiva apareció un efecto significativo del rol sexual de los sujetos, verificándose las comparaciones planeadas.

La segunda situación intentaba evocar simpatía y apoyo al escuchar a un sujeto, sin que se tuviera que asumir ningún tipo de iniciativa. De nuevo se procedió con un análisis de varianza sexo $\times$ rol y se establecieron una serie de comparaciones ortogonales: a) los sujetos femeninos y andróginos serán más afectivos que los sujetos masculinos y b) los sujetos femeninos y andróginos no diferirán entre sí.

Los resultados verificaron todas las hipótesis, aun en el caso de que no se distinguiera entre andróginos e indiferenciados. Un reanálisis efectuado separando ambos grupos puso de manifiesto que no había diferencias consistentes entre ambos. 
Por lo tanto, Bem concluyó, a la vista de estos resultados, que la baja expresividad de la mujer femenina sólo se da en tareas muy concretas y en las que pueden intervenir otros factores de importancia como, por ejemplo, si le gustan o no los animales. Sólo los sujetos andróginos son capaces de ser instrumentales y expresivos, dependiendo de la situación, mientras que los sujetos tipificados sexualmente parecen restringir su comportamiento a cualquiera de estos dominios congruentes con su sexo.

En la misma dirección que sus estudios anteriores, Bem y Lenney (1976) realizaron otro experimento con el objeto de ofrecer todavía mayor evidencia a la hipótesis de la restricción comportamental de los sujetos tipificados sexualmente. En esta ocasión se trataba de comprobar no sólo que los sujetos tipificados (en este caso, hombres masculinos y mujeres femeninas) evitaban activamente comportamientos de género cruzado (es decir, los hombres masculinos evitarían los comportamientos femeninos y las mujeres femeninas los comportamientos masculinos), sino también que éstos eran motivacionalmente problemáticos, y que su realización conllevaría un cierto «disconfort» psicológico y una merma de su autoestima.

Más concretamente, para averiguar si los sujetos tipificados preferían actividades apropiadas a su sexo en mayor medida que los sujetos andróginos -incluso en situación donde estas preferencias podían tener algún tipo de coste adicional- se le entregó una lista de sesenta actividades, presentadas por pares, y se les pidió que eligiesen una de cada par, la que estuvieran dispuestos a realizar, a partir de la cual se haría una fotografía. Cada actividad tenía asignada una pequeña cantidad de dinero, de tal manera que las menos apropiadas al sexo del sujeto eran las más pagadas. Después de realizar tres conductas masculinas, tres femeninas y tres neutras se daba por finalizado el experimento. Tras cada una de estas actividades, el sujeto contestaba a una serie de preguntas para determinar cómo se había sentido al realizarlas.

El análisis de varianza realizado con las puntuaciones extraídas de la lista de conductas elegidas mostró un efecto significativo del rol del sujeto, en el sentido de que los sujetos tipificados sexualmente eran significativamente más estereotipados en sus elecciones que los sujetos andróginos y de género cruzado (hombres femeninos y mujeres masculinas). Además estos dos grupos últimos no difirieron entre sí.

Para averiguar si los individuos tipificados sexualmente experimentaron el mayor grado de «disconfort» psicológico, de nuevo se realizó un análisis de varianza y una serie de comparaciones planeadas. Los resultados indicaron que los sujetos tipificados sexualmente se sintieron significativamente peor después de realizar actividades de género cruzado que los andróginos y los sujetos de género invertido. Estos dos últimos, además, no difirieron entre sí.

La hipótesis de Bem sobre la flexibilidad comportamental de los sujetos andróginos ha sido retomada y puesta a prueba relativamente en pocas ocasiones. Aunque sus trabajos están realizados en el laboratorio, otros intentos por acercarse a esta cuestión han optado por la perspectiva correlacional (Orlofsky y Windel, 1978; Wiggins y Holzmuller, 1978, 1981; Anderson, 1986).

Algunos intentos de réplica han variado sustancialmente el procedimiento seguido, por lo que sus resultados deben verse con cautela (Helmreich, Spence y Holahan, 1979; Heilbrun y Pitman, 1979). Otros trabajos son aproximaciones al tema de la flexibilidad pero desde vertientes bastante diferentes: Ickes y Barnes (1978), en situaciones de interacción no estructurada; Kelly, O'Brien y Hosford (1981), en situaciones que requerían asertividad reforzante o de rechazo; Ho (1981), basándose en la habilidad para solucionar problemas en niños; La France y Carmen (1980) mediante el estudio de la co- 
municación no verbal. Otras investigaciones hablan en favor de la interacción entre rol y situación (Klein y Willerman, 1979; Alagna, 1982).

El objetivo de este trabajo ha sido precisamente el de estudiar el tema de la adaptabilidad comportamental desde una perspectiva más amplia y rigurosa (Sebastián, 1986), aunque en esta ocasión nos ceñiremos a las hipótesis y resultados que directamente replican los hallazgos de Bem ${ }^{1}$.

Sin embargo, nuestra investigación no puede ser considerada como una copia fidedigna de la realizada por esta autora. Nuestro intento ha introducido algunas variaciones importantes como son: a) el unificar en un único experimento todas las hipótesis y planteamientos de los tres trabajos de Bem y colaboradores, $b$ ) la inclusión del grupo del rol sexual indiferenciado en los análisis desde el comienzo, lo cual tiene implicaciones en el porcentaje de sujetos asignados a cada uno de los roles sexuales, y $c$ ) el variar la naturaleza de las tareas objeto de análisis posterior y el grado de estructuración de la situación.

En cualquier caso, a nosotros nos ha interesado hacer recaer el centro de la investigación en los sujetos andróginos, además de tener en cuenta las hipótesis de Bem.

Por tanto, trataremos de comprobar que los individuos andróginos a) mostrarán una mayor implicación en tareas tanto masculinas como femeninas, $b$ ) preferirán en menor medida actividades apropiadas a su sexo y c) experimentarán un mayor bienestar psicológico al desarrollar este tipo de actividades que cualquier otro grupo de rol sexual.

\section{METODO}

\section{Sujetos}

En el experimento participaron 48 sujetos ( 24 hombres y 24 mujeres), todos ellos estudiantes de primero y segundo curso de Psicología de la Universidad Autónoma de Madrid. Los sujetos se repartían atendiendo a su rol sexual del siguiente modo: tanto en el grupo de los hombres como de mujeres, ocho sujetos eran masculinos, ocho femeninos, ocho andróginos y ocho indiferenciados. El rango de edad oscilaba entre dieciocho y veinticinco años.

Estas personas fueron seleccionadas al azar dentro de su grupo, a partir de una muestra de 201 estudiantes de psicología, a quienes en la primavera de 1985 se les administró el BSRI.

El rol del sujeto se estableció por el método de la mediana (Bem, 1979; Spence y cols., 1979). En la muestra de 201 sujetos ( 63 hombres y $138 \mathrm{mu}$ jeres) la Mediana de la escala de Masculinidad fue de 85,00 y la de Feminidad de 91,28 .

\section{Instrumentos}

El BSRI es un inventario de autoinforme que consta de 60 características de personalidad, 20 masculinas, 20 femeninas y 20 neutras. Requiere del sujeto que indique con qué exactitud le describe cada una de las características a partir de una escala de siete puntos (para más información ver Ben, 1974).

- Rejillas de observación.

Ya que una parte del experimento trataba de averiguar si existían diferencias en cuanto al grado de implicación de los sujetos según su rol sexual en tareas masculinas y femeninas, nos vimos en la obligación de construir, 
una vez elegidas una conducta de cada tipo, una rejilla de observación para cada una de ellas con vistas a poder recoger la información pertinente.

Estas rejillas fueron construidas poco a poco a partir de la categorización de unidades de observación, puestas a prueba en varios grupos de voluntarios y en un grupo preexperimental. Además, fueron incluidas otras conductas cuya realización significaba una elevada implicación del sujeto en la tarea.

La elección de la conducta masculina y de la conducta femenina objeto de observación no fue fácil. Algunas reticencias nos hicieron desechar el azar como método de elección debido a la heterogenidad de las conductas extraídas (ver siguiente epígrafe), y a que creíamos que deberíamos controlar una serie de aspectos que podrían influir a la hora de su realización. Por todo ello, pusimos a prueba varias de éstas para estudiar sus posibilidades de medición y su mayor pertinencia. Finalmente, escogimos la conducta de «limpiar una escopeta de caza» como conducta masculina, y «colocar flores en un jarrón» como conducta femenina.

Siguiendo el desarrollo de los experimentos de Bem y colaboradores, fueron elaborados otros dos instrumentos de recogida de datos: un índice del grado de implicación evaluado por parte de los experimentadores, en una escala de cinco puntos, y una hoja de registro de comentarios.

\section{Validación de las tareas experimentales como estereotipicamente masculinas o femeninas}

Con el fin de identificar las conductas masculinas, femeninas y neutras que nos servirían para desarrollar nuestra investigación, se ideó un conjunto de 165 conductas simples, cotidianas que, en opinión de siete jueces, podían ser conceptualizadas como tales.

En orden a validar estas conductas como estereotípicamente masculinas, femeninas o neutras, fueron presentadas a 40 jueces (20 hombres y 20 mujeres), estudiantes de cuarto y quinto curso de psicología, para que evaluasen su grado de masculinidad o feminidad en la sociedad española. A los jueces se les recalcó que no se les pedía su opinión personal sino que especificasen en una escala que iba de -3 (muy masculina) a +3 (muy femenina) cómo eran concebidas cada una de ellas por la sociedad española.

Las respuestas de los jueces fueron analizadas a partir del procedimiento seguido por Bem (1975b) aunque algo más restrictivo. El criterio de selección de las conductas masculinas fue que deberían ser evaluadas por el 70 por 100 de los jueces como bastante o muy masculinas y no fueran evaluadas por ningún juez como femeninas (ya fuese algo, bastante o muy femenina). Debido a que este criterio era respetado por una treintena de conductas, debimos afinar todavía más para seleccionar entre ellas. Para ello atendimos al valor escalar (mediana) y a la dispersión discriminante o ambigüedad de los items, en el sentido de ordenar las conductas en base a la proximidad de su mediana con el valor 1 ó 7 , y que la distancia intercuartílica $\left(Q_{3}-Q_{1}\right)$, a partir de la cual puede obtenerse el coeficiente de ambigüedad, fuese pequeña, lo cual informa de la poca dispersión de los juicios.

Las conductas masculinas (boxear, afeitarse, limpiar las bujías de un coche, marcar el paso y saludar militarmente, cortar leña, levantar pesas y estirar tensores, etc.) y femeninas (ponerse rulos y horquillas en el pelo, andar con zapatos de tacón alto, hacerse la manicura, adoptar una posición sexy, maquillarse, bordar, etc.), elegidas hasta un total de dieciséis de cada tipo, no sólo cumplían este criterio sino que además el 80 por 100 de los jueces las calificaban de bastante o muy masculinas o femeninas, según el caso. 
Las conductas neutras fueron seleccionadas con arreglo al siguiente criterio: que fueran evaluadas por el 70 por 100 de los jueces como neutra (punto medio de la escala), y ninguno de ellos la evaluase como bastante o muy masculina o femenina. Posteriormente, también se tuvo en cuenta su proximidad al valor neutro y su distancia intercuartílica. De nuevo fueron escogidas dieciséis conductas (corregir faltas de ortografía, completar letras, resumir, lavarse los dientes, buscar un teléfono, tocar la guitarra, contar, etc.), de las cuales diez cumplían el criterio de acuerdo de los jueces del 80 por 100 .

\section{Cuestionario de elección de conductas}

Una vez obtenidas las dieciséis conductas de cada tipo, se procedió a distribuirlas en pares conflictivos, donde variaba el tipo de conducta y pares no conflictivos, donde las dos conductas eran del mismo tipo (ambas masculinas, femeninas o neutras). En los pares conflictivos las conductas apropiadas sexualmente se pagaban menos y las no apropiadas se pagaban más. En los pares no conflictivos la elección de la conducta que se pagaba más era realizada aleatoriamente. En cuanto al dinero, el sujeto podía ganar como máximo 129 pesetas y como mínimo 84 pesetas. Como hizo Bem, el dinero asignado a todas las actividades variaba de 2 a 6 unidades, mientras que dentro de cada par la diferencia oscilaba entre 1 y 3 unidades. Los pares de control servían también para camuflar el verdadero propósito del experimento.

Por tanto, hubo que disponer de dos cuestionarios de elección de conductas, uno para varones y otro para mujeres, cada uno de ellos con sus pagos diferenciales correspondientes.

Aunque el sujeto elegía una conducta de cada par, en el laboratorio sólo tenía que desarrollar tres masculinas, tres femeninas y dos neutrales, como veremos más adelante. La elección de estas conductas y su orden fue decidido en parte por azar y en parte por criterios racionales.

\section{Cuestionario de Bienestar psicológico}

Bem y Lenney (1976) desarrollaron una escala de «disconfort psicológico» para medir cómo se habían sentido los sujetos al realizar las tareas. En este mismo orden de cosas, nosotros también les preguntábamos cómo se habían sentido, inmediatamente después de completar cada actividad. A partir de una escala de siete puntos, se le pedía al sujeto que evaluase lo divertido, agradable, atractivo, masculino/femenino, extraño que se había sentido, o si lo había realizado en tensión. También se le preguntaba al sujeto si tenía experiencia y con qué frecuencia había realizado esa actividad.

\section{Procedimiento}

Los sujetos experimentales recibieron en sus domicilios una carta en la cual se les explicaba que habían sido elegidos para la primera parte de un proyecto de investigación, por lo cual se les convocaba un día y a una hora determinada.

Al comienzo de cada una de las sesiones los sujetos eran recibidos por una recepcionista, y les informaba que el objetivo de la investigación era determinar si las personas emitían juicios diferentes sobre la personalidad de un individuo cuando veían a éste realizando distintas actividades. Por consiguiente, lo que necesitábamos era precisamente una misma persona desarro- 
llando diferentes actividades, y era el sujeto quien podría proporcionarnos este material, mediante la filmación en video de distintas situaciones.

A continuación se le explicaba que le daríamos una lista con 48 actividades presentadas por pares, y que debía escoger una de cada par, la que creía que podía representar realmente por un breve espacio de tiempo (3-5 minutos). Debajo de cada par se encontraría una pequeña cantidad de dinero. Al final se sumaría el dinero de cada conducta y se le pagaría al concluir el experimento.

La diferencia de pago entre las actividades se debía a que había una serie de conductas de las cuales teníamos muy pocas filmaciones y, por ellas pagaríamos un poco más de dinero.

Se puso especial énfasis en transmitir al sujeto que lo que nos interesaba no era lo bien que desarrollase cada actividad o si renía experiencia, sino que quisiera hacerla y se sintiera implicado, para que la escena fuese lo más convincente posible. También se informaba de que cada una de estas actividades tendría unas instrucciones simples escritas.

Cuando entraba en el laboratorio era recibido por los dos experimentadores (un hombre y una mujer). En todos los casos, el experimentador principal era de distinto sexo que el sujeto experimental, y era realmente el que llevaba a cabo las presentaciones. El otro era el encargado de filmar y así se le comunicaba al sujeto. Los dos experimentadores fueron los mismos para los 48 sujetos.

Una vez que el sujeto había elegido las conductas que estaba dispuesto a llevar a cabo, el experimentador - después de aparentar buscar la tarjeta explicativa de las conductas elegidas - le informaba que como ya teníamos muchas filmaciones de las que había elegido, sólo iba a desarrollar ocho. Podría ocurrir que alguna de ellas no la hubiera elegido, aunque esto lo intentaríamos evitar siempre que fuera posible.

Si èl sujeto había entendido la dinámica de la sesión se comenzaba con la primera tarea, leyendo previamente la tarjeta donde había unas instrucciones simples escritas. El orden de realización de las tareas fue el siguiente: tarea neutra (escogida por el sujeto), tarea masculina (escogida por el sujeto), femenina fija (colocar flores en el jarrón), femenina escogida, masculina fija (levantar pesas y estirar tensores), neutra escogida, femenina fija (ponerse rulos y horquillas en el pelo) y masculina fija (limpiar una escopeta de caza).

Después de realizar cada una de estas tareas se le ofrecía al sujeto un cuestionario para que evaluase cómo se había sentido al realizar cada una de ellas.

Los experimentadores observaban atentamente el desarrollo de las conductas objeto de observación, y evaluaban la actuación del sujeto, como ya se puso de manifiesto anteriormente. Estas conductas clave (colocar flores en un jarrón y limpiar una escopeta de caza) eran observadas al otro lado de un espejo unidireccional que había en el laboratorio por dos personas (observadores independientes), mediante las rejillas elaboradas para tales situaciones. Los observadores poseían suficiente práctica de observación y habían realizado un período de adiestramiento. El índice de acuerdos obtenido fue del 96 por 100 . Los comentarios eran registrados por otro ayudante situado en la misma sala detrás de unos cubos de madera que impedían poder verlo. Por supuesto, ninguna de las personas que trabajaron en este experimento sabían el rol sexual de los sujetos experimentales.

Una vez terminado el experimento, se le agradecía al sujeto su colaboración y después de informarle que lo que había sucedido en el laboratorio era confidencial, se le acompañaba hasta la recepcionista, quien pagaba al sujeto por las conductas elegidas y le pasaba un pequeño cuestionario que recogía información sobre cuestiones acerca del experimento. 


\section{RESULTADOS}

Antes de proceder al análisis de resultados conviene señalar dos cuestiones referidas a los mismos. En primer lugar, ya que parte de la obtención de los datos se ha basado en el procedimiento de observación, mediante códigos de categorías de conducta (Fernández Ballesteros, 1983), conviene precisar que los desacuerdos entre los dos jueces han sido desestimados. Así, pues, la variable implicación ha quedado definida operativamente como el número de conductas orientadas a la tarea, con lo cual, la puntuación total en implicación de un sujeto en una tarea determinada es igual al número de veces que ha realizado las conductas presentes en la rejilla de observación.

En segundo lugar, antes de proceder al análisis de la puntuación de bienestar, calculamos el coeficiente alfa de consistencia interna de todas las respuestas, obteniendo el siguiente resultado: $\alpha=0,81$.

Para comprobar si las medias de los distintos grupos de rol sexual diferían significativamente en cuanto a su implicación én cada una de las tareas seleccionadas, fue realizado un análisis de varianza cuyos resultados pueden verse en la Tabla 1.

\section{TABLA 1}

Implicación media de los distintos grupos de rol sexual en las tareas masculina y femenina

\begin{tabular}{lcccccc}
\hline & Masculinos & Femeninos & Andróginos & Indiferenciados & F & Significación \\
\hline Flores & 21.250 & 34.167 & 32.083 & 28.033 & 7,07 & 0,0006 \\
Escopeta & 18.167 & 12.417 & 18.667 & 14.167 & 4,51 & 0,0076 \\
\hline
\end{tabular}

En la tarea femenina de «colocar flores en un jarrón» hubo un efecto principal significativo del rol del sujeto $(p<0,001)$. Las comparaciones planeadas indicaron que, como anticipábamos, había diferencias significativas entre los sujetos masculinos y femeninos $(\mathrm{t}(44)=-4,28 \mathrm{p}<0,001)$, en el sentido de que los sujetos femeninos y andróginos se implicaron más en esta tarea que los sujetos masculinos. Todos los niveles de probabilidad están basados en las pruebas de significación de dos colas.

En la tarea masculina de «limpiar una escopeta de caza» también hubo un efecto principal significativo del rol del sujeto $\mathrm{p}<0,01$ ). Las diferencias significativas encontradas entre los distintos grupos del rol sexual también cumplían las previsiones. Las comparaciones planeadas dieron como resultado que los sujetos masculinos y femeninos diferían significativamente en su grado de implicación $(t(44)=3,04 \mathrm{p}<0,05)$, en el sentido de que los primeros tuvieron mayores puntuaciones que los segundos. También aparecieron diferencias entre los sujetos femeninos y andróginos $(t(44)=-2,82 \mathrm{p}<0,05)$, a favor de estos últimos.

Por tanto, nuestra hipótesis parece confirmarse ya que sólo los sujetos andróginos fueron capaces de implicarse realmente tanto en tareas masculinas como femeninas y, como se esperaba, estuvieron al mismo nivel que los sujetos masculinos en la tarea masculina, y que los sujetos femeninos en la tarea femenina.

Los sujetos indiferenciados ocuparon el tercer lugar en cuanto a las medias en implicación tanto en la tarea masculina como femenina. En la primera, aparecieron diferencias significativas entre los sujetos indiferenciados y masculinos $(t(44)=2,16 \mathrm{p}<0,05)$ e indiferenciados y andróginos 
$(t(44)=2,18 p<0,05)$. En cuanto a la tarea femenina hubo diferencias significativas entre sujetos masculinos e indiferenciados $(t(44)=-3,1 \mathrm{p}<0,01)$ y femeninos e indiferenciados $(t(44)=2,58 p<0,05)^{2}$.

Para saber la influencia de las puntuaciones en la escala de Masculinidad y Feminidad del BSRI en las variables estudiadas por nosotros, se realizó posteriormente un análisis de varianza de dos factores masculinos $\times$ femeninos. Los resultados del análisis mostraron que para la tarea de «colocar flores en un jarrón», ambas variables tuvieron un efecto significativo (para masculinidad $F(1,44)=4,61 p<0,05$ y para feminidad $F(1,44)=15,23 p<0,001)$. Las comparaciones entre los distintos grupos revelaron diferencias significativas entre los sujetos femeninos y masculinos $(\mathrm{t}(44)=4,28 \mathrm{p}<0,001)$ y entre los masculinos y andróginos $(\mathrm{t}(44)=4,28 \mathrm{p}<0,01$ debido a las altas puntuaciones en feminidad de los individuos femeninos y andróginos, fundamentalmente.

Algo parecido sucedió en cuanto a la implicación en «limpiar una escopeta de caza». En este caso, sólo hubo un efecto significativo de la masculinidad $(F(1,44)=12,86 p<0,001)$. Las diferencias entre los distintos grupos justamente se establecieron entre aquellos que diferían en su grado de masculinidad: sujetos masculinos y femeninos $(t(44)=-3,04 p<0,05)$, y femeninos y andróginos $(\mathrm{t}(44)=2,82 \mathrm{p}<0,05)$.

En este mismo sentido, el análisis de regresión efectuado con el objeto de conocer la contribución de las puntuaciones de masculinidad y feminidad -más que su función predictiva - en la variable implicación, mostró, como puede verse en la Tabla 2, unos resultados concordantes con los anteriores, si bien nos informaron del coeficiente de correlación negativo existente entre las puntuaciones de masculinidad y el desempeño en la tarea de las flores, tanto para hombres como para mujeres, y entre las puntuaciones de feminidad y la tarea de la escopeta, en este caso sólo en el grupo de los hombres.

TABLA 2

Resultados del análisis de regresión

\begin{tabular}{lrll}
\hline & Hombres & Mujeres & Total \\
\hline Flores: & & & \\
Masculino & $8,16 \%(\mathrm{r}=-0,28)^{*}$ & $24,09 \%(\mathrm{r}=-0,49)^{* *}$ & $14,58 \%(\mathrm{r}=-0,38)^{* *}$ \\
Femenino & $15,39 \%(\mathrm{r}=-0,37)^{*}$ & $14,98 \%(\mathrm{r}=-0,36)^{* *}$ & $14,41 \%(\mathrm{r}=, 0,36)^{* *}$ \\
Total & & & $28,99 \%$ \\
& & & \\
Escopeta: & & & \\
Masculino & $16,60 \%(\mathrm{r}=0,40)^{*}$ & $40,08 \%(\mathrm{r}=0,63)^{* * *}$ & $31,69 \%(\mathrm{r}=0,56)^{* * *}$ \\
Femenino & $3,50 \%(\mathrm{r}=-0,16)$ & $0,23 \%(\mathrm{r}=0.08)$ & $0,23 \%(\mathrm{r}=0,02)$ \\
Total & & & $31,92 \%$ \\
\hline
\end{tabular}

Por lo tanto, tanto la feminidad y la masculinidad fueron importantes en el grado de implicación en la conducta de «colocar flores en un jarrón», aunque esta última con signo negativo, y sólo la masculinidad fue importante en la conducta de «limpiar una escopeta de caza».

En cuanto a la segunda variable estudiada - las preferencias comportamentales-, los resultados del análisis de varianza llevado a cabo mostraron un efecto principal significativo del rol del sujeto $(F(6,771)=2,50 \mathrm{p}<0,05$. Las comparaciones entre los distintos grupos pusieron de manifiesto diferencias significativas únicamente entre los sujetos tipificados (hombres masculinos y mujeres femeninas) y los de género cruzado (hombres femeninos y mu- 
jeres masculinas $)(F(19,62)=3,20 \mathrm{p}<0,05)$, en el sentido de que los primeros eligieron un mayor número de conductas apropiadas.

Por consiguiente, con respecto a las hipótesis de Bem podemos decir que: los sujetos tipificados sexualmente aunque difirieron significativamente de los sujetos de rol invertido, no lo hicieron de los sujetos andróginos e indiferenciados, si bien, existió una tendencia significativa entre los tipificados y los andróginos $(p=0,06)$, en el sentido de que los primeros escogieron mayor número de conductas apropiadas a su sexo que los segundos (ver Tabla 3 ).

TABLA 3

Puntuaciones medias en preferencias y bienestar de los distintos grupos de rol sexual

\begin{tabular}{lcccccc}
\hline & Tipificados & Género cruzado & Andróginos & Indiferenciados & F & Significación \\
\hline Preferencias & 7.833 & 5.667 & 6.417 & 7.167 & 3,59 & 0,02 \\
Bienestar & 3,09 & 3,77 & 4,01 & 3,66 & 2,24 & 0,05 \\
\hline
\end{tabular}

Con respecto a la tercera variable que se refería al bienestar o «confort psicológico» experimentado tras la realización de conductas no apropiadas sexualmente, se encontró que sólo los sujetos andróginos diferían significativamente de los tipificados sexualmente $(t(22)=2,45 p<0,05)$, sin-que esto se pueda decir con respecto a los sujetos de género cruzado e indiferenciados. Por tanto, la hipótesis de Bem sólo se ha visto parcialmente confirmada.

Tanto en este caso como en el anterior, no hubo diferencias significativas entre los sujetos andróginos, indifèrenciados y de rol invertido, ital y como hipotetizó Bem.

$\mathrm{El}$ análisis de varianza de dos factores (Masc $\times \mathrm{Fem}$ ) para la variable bienestar, descubrió la existencia de efectos significativos de la masculinidad $(\mathrm{F}(1,44)=20,53, \mathrm{p}<0,0001)$, de la feminidad $(\mathrm{F} 01,44)=9,09 \mathrm{p}<0,01)$ y de la interacción $F(1,44)=6,47 p<0,05)$ en cuanto al grado de bienestar expresado por los sujetos en la tarea de la escopeta. Fueron encontradas diferencias significativas entre los grupos indiferenciados y femeninos $(t(44)=3,93 \mathbf{p}<0,01)$, femeninos y masculinos $(t(44)=-5,34 \mathrm{p}<0,001)$ y femeninos y andróginos $(t(44)=-5,00 p<0,001)$, siempre expresando un menor bienestar los sujetos con alta feminidad. Ningún otro resultado fue significativo, si bien hubo una tendencia $(p=0,078)$ a la que las puntuaciones en feminidad fueran significativas en cuanto al grado de bienestar experimentado en la tarea de las flores.

Una vez realizados los análisis de varianza de un factor, se procedió a efectuar análisis de dos factores Rol × Sexo. En la conducta de «colocar flores en un jarrón» se encontró un efecto principal significativo del rol del sujeto $(F(3,40)=6,57 p<0,001)$, con las mujeres femeninas en un extremo y las mujeres masculinas en el otro. En la tarea de «limpiar una escopeta de caza» también se descubrió un efecto principal significativo del rol del sujeto $(F(3,40)=4,67 p<0,01)$, con los hombres masculinos en un extremo y las mujeres indiferenciadas y femeninas en el otro. En este caso, hubo asimismo una tendencia significativa a que el sexo del sujeto fuese significativo $(p=0,069)$.

En lo que respecta a las preferencias conductuales, hubo un efecto significativo de la interacción ( $\mathrm{Rol} \times$ Sexo) en el número de conductas elegidas que fuesen apropiadas sexualmente, con los hombres masculinos y las mujeres andróginas en los extremos superior e inferior respectivamente $(F(3,40)=3,64$ $\mathrm{p}<0,05)$. 
Unicamente en la tarea de la escopeta fue hallado un efecto significativo del rol del sujeto en la variable «bienestar», siguiendo con este tipo de análisis $(F(3,40)=11,82 \mathrm{p}<0,0001)$. Las comparaciones entre los distintos grupos arrojó diferencias significativas entre las mujeres femeninas y casi todos los demás grupos: hombres masculinos y mujeres femeninas ( $p<0,01)$, mujeres masculinas y mujeres femeninas $p<0,001$ ), hombres andróginos y mujeres femeninas $(p<0,01)$, hombres indiferenciados y mujeres femeninas $(p<0,05)$ y mujeres indiferenciadas y mujeres femeninas $(p<0,05)$.

También se llevaron a cabo otras series de análisis complementarios de los anteriores. En este sentido cabe mencionar el análisis de los efectos de covariación de la práctica o experiencia que podría tener el sujeto en este tipo de tareas. En la tarea de las flores no hubo ningún efecto significativo de la experiencia que pudiera tener la persona, aunque sí hubo una tendencia significativa de la misma $(\mathrm{p}=0,09)$. En cambio, en la conducta de la escopeta sí se encontró ùn efecto significativo de la experiencia en esta actividad $(p<0,05)$. No obstante, em ambos casos una vez eliminado el efecto de la práctica, el rol deYsujeto siguió siendo significativo $(p<0,001$ en flores y $p<0,05$ en escopeta). En lo que se refiere al grado de bienestar experimentado por el sujeto al realizar estas actividades, no se encontró un efecto significativo de la experiencia previa.

Con respecto al dinero que los sujetos obtenían por sus elecciones conductuales o preferencias, no se encontraron diferencias significativas entre los distintos grupos de rol sexual, ni entre los sexos, si bien, fueron los sujetos con un rol masculino los que se llevaron más cantidad. La media por sujeto fue de 88 pesetas.

Unicamente hubo una tendencia significativa $(p=0,07)$ dentro del grupo de las mujeres, a que las andróginas y masculinas sumaran más cantidad que las femeninas e indiferenciadas, que a su vez no diferían entre sí.

Tampoco hubo diferencias significativas entre los distintos grupos en cuanto al número de conductas de bajo pago escogidas en los pares no conflictivos.

En cuanto a las puntuaciones de los experimentadores, al análisis de varianza no paramétrico (Kruskal-Wallis) realizado señaló que en la tarea de la escopeta los sujetos andróginos obtuvieron significativamente mayores puntuaciones $(p<0,01)$.

También fue hallado en el análisis de los datos un efecto significativo del rol del sujeto $(p<0,05)$ y de la interacción $\operatorname{Rol} \times$ Sexo $(p<0,05)$ en cuanto al número de acuerdos obtenidos en la evaluación realizada por los experimentadores, siruándose las mujeres indiferenciadas en el extremo de mayor similitud en los juicios de éstos, y los hombres femeninos los que alcanzaron menor acuerdo.

Merece la pena exponer a continuación algunos de los resultados obtenidos en la contestación a la pregunta de si se habían sentido masculinos/femeninos (dependiendo del sexo) al realizar cada una de las tareas (esta pregunta iba incluida en el Cuestionario de Bienestar).

En la conducta de las flores, las mujeres andróginas se sintieron las más femeninas, mientras que los hombres indiferenciados se sintieron los menos masculinos $(\mathbf{p}<0,01)$. En cuanto a la tarea de la escopeta, los hombres masculinos se sintieron los más masculinos al realizarla y las mujeres femeninas las menos femeninas ( $p=0,08$ para el sexo y $p=0,09$ para el rol).

En general, es posible extraer un curioso patrón de resultados teniendo en cuenta todas las conductas masculinas y femeninas realizadas por los sujetos, y no solamente las tareas blanco de observación: las mujeres andróginas fueron las que se sintieron más femeninas en todas las tareas femeninas, 
mientras que en las tareas masculinas, las mujeres femeninas fueron siempre las que se sintieron menos femeninas.

Por último, comentaremos algunos de los datos provenientes del cuestionario que se les pasaba a los sujetos una vez terminado el experimento. El 25 por 100 de las personas consideraron el experimento interesante, el 46 por 100 entretenido, divertido y original, y el 6,3 por 100 lo consideró extraño o raro. El 37,5 por 100 de los sujetos se creyeron lo que se les había dicho sobre la finalidad del instrumento, el 12,5 por 100 acertaron más o menos el objetivo real. No les molestó nada durante el experimento al 85,5 por 100 de los sujetos. No les gusţó desarrollar las tareas no escogidas al 4,2 por 100 . En líneas generales la colaboración de los sujetos fue excelente.

\section{DISCUSION}

A partir de los resultados obtenidos podemos comprobar que las hipótesis de Bem han sido casi confirmadas totalmente. En lo que respecta a la variable implicación, los resultados muestran que realmente sólo los sujetos andróginos fueron capaces de realizar bien tanto la tarea masculina como la femenina. También, como se esperaba, los sujetos masculinos se implicaron convenientemente sólo en la tarea masculina, mientras que los femeninos lo hicieron sólo en la tarea femenina.

Además, no han aparecido diferencias significativas entre los sujetos andróginos y masculinos en la tarea de limpiar una escopeta de caza, y entre andróginos y femeninos en colocar flores en un jarrón, cumpliéndose de este modo las previsiones que hicimos al respecto.

En cuanto a los sujetos indiferenciados, parecen colocarse en el tercer lugar en cualquiera de las dos tareas. Debido a que en la tarea masculina se encontraron diferencias significativas entre andróginos e indiferenciados, creemos que es necesario diferenciar - en cuanto a la polémica suscitada en la literatura con respecto a este punto- entre aquellos sujetos con altas puntuaciones en $\mathrm{M}$ y $\mathrm{F}$ (andróginos), y los que obtienen bajas puntuaciones en ambas escalas (indiferenciados).

Por consiguiente, nuestro trabajo parece confirmar los mismos resultados que Bem (Bem, 1976; Bem, Martyna y Watson, 1976) obtuvo en un experimento de similares características, si bien, en nuestro caso pusimos a prueba conductas que no elicitaban directamente comportamientos recogidos en el BSRI, ni rasgos instrumentales y expresivos, así que las críticas de Spence y Helmreich (1979) parecen irrelevantes, ya que las predicciones realizadas con este instrumento parecen ir más allá del campo circunscrito a la expresividad e instrumentalidad.

En cuanto a las hipótesis referidas a la mayor preferencia del rol sexual andrógino por conductas no apropiadas sexualmente y a un mayor bienestar (o menor «disconfort» psicológico en palabras de Bem) al realizar éstas, los datos muestran que en lo sustancial parecen haberse verificado.

Más concretamente, los sujetos de género cruzado y andróginos (aunque en estos últimos hubo una tendencia altamente significativa) ${ }^{4}$ difirieron de los sujetos tipificados sexualmente en cuanto al número de conductas elegidas que eran inapropiadas al sexo del individuo. En la variable Bienestar sólo se encontró una diferencia significativa, precisamente entre sujetos andróginos y tipificados sexualmente.

No hubo diferencias entre sujetos andróginos, de género cruzado e indiferenciados en ambas variables, tal y como predijo Bem (Bem y Watson, 1976). Sin embargo, al establecerse diferencias entre sujetos andróginos y ti- 
pificados y no hacerlo entre indiferenciados y tipificados, conviene seguir considerándolos por separado.

En resumen, de nuevo parece que es el rol sexual andrógino el que siente un mayor grado de bienestar al realizar conductas que son sancionadas socialmente como inapropiadas a su sexo, y el que escoge un mayor número de este tipo de comportamientos.

El único dato contrario a los esperado, según las hipótesis establecidas por Bem, fue que los sujetos de género cruzado no difirieron de los tipificados sexualmente en la variable Bienestar.

Finalmente, sólo queda concluir que la restricción comportamental de los sujetos no andróginos (fundamentalmente de los sujetos tipificados sexualmente) parece evidente a tenor de los resultados obtenidos tanto en el trabajo de Bem como en el nuestro y que, por tanto, puede ser defendida la mayor flexibilidad comportamental de los sujetos pertenecientes a este rol sexual. Por otra parte, también parece haber quedado respaldada la validez predictiva del BSRI.

Sin embargo, un mayor cúmulo de investigación debe ser realizada en este tema, con el fin de clarificar y profundizar en el estudio sistemático de la influencia de las variables sexo, rol y su interacción, tal y como se ha llevado a cabo en otro lugar (Sebastián, 1986).

\section{Notas}

1 Las hipótesis originales ascienden a veinticuatro.

2 Estos resultados de los sujetos indiferenciados fueron extraídos del paquete estadístico SPSS. Los demás fueron realizados con BMDP.

${ }^{3}$ Creemos que esta afirmación puede ser hecha, aunque con prudencia, debido a que la significación fue $p=0,06$.

\section{Referencias}

ALAGNA, S. W. «Sex role identity, peer evaluation of competition, and the responses of women and men in a competitive situation». Journal of Personality and Social Psychology, 1982, 43, 546-554.

ANDERSON, K. L. "Androgyny, flexibility and individualism». Journal of Personality Assessment, 1985, 50, 265-278.

BaKan, D. The duality of human existence. Chicago: Rand McNally, 1966.

BAUCOM, D. H. "Independent masculinity and femininity scales on the California Psychological Inventory». Journal of Consulting and Clinical Psychology, 1976, 44, 876.

BEM, S. L. «The measurement of psychological androgyny». Journal of Consulting and Clinical Psychology, 1974, $42,155-162$.

BEM, S. L. «Androgyny and mental health». Paper presented at the meeting of the American Psychological Association, Chicago, septiembre de 1975.

BEM, S. L. «Sex-role adaptability: one consequence of psychological androgyny». Journal of Personality and Social Psychology, 1975b, 31, 634-643.

BEM, S. L. «Theory and measurement of androgyny: a reply to the Pedhazur-Tetenbaum and Locksley-Colten critiques». Journal of Personality and Social Psycbology, 1979, 37, 1047-1054.

BEM, S. L., y LENNEY, E. «Sex-typing and the avoidance of cross-sex behavior». Journal of Personality and Social Psychology, 1976, 33, 48-54.

BEM, S. L.; MARTYNA, W., y WATSON, C. «Sex-typing and androgyny: further explorations of the expressive domain». Journal of Personality and Social Psychology, 1976, 34, 1016-1023.

BerzINS, J. I., y WELLING, M. A., WETTER. R. E. The PRF-Andro Scale user's manual. University of Kentucky, 1978.

CONSTANTINOPLe, A. «Masculinity-feminity: an exception to a famous dictum?» Psychological Bulletin, 1973, $80,389-407$.

ERIKSSON, E. H. «Inner and outer space: reflections on womanhood», en R. J. Lifton (ed.): The woman in America. Boston: Houghton Mifflin, 1964.

FERnANDEZ BALlesteros, R. «Técnicas de observación», en R. Fernández Ballesteros (dir.): Psicodiagnóstico (Vol. I), Madrid: UNED, 1983.

FERNANDEZ SANCHEZ, J. Nuevas perspectivas en la medida de la masculinidad y feminidad. Tesis doctoral. Madrid: Universidad Complutense, 1983. 
Heilbrun, A. B., y PiTman, D. «Testing some basic assumptions about psychological androgyny. Journal of Genetic Psychology, 1979, 135, 175-188.

Helmreich, R. J.; SPEnCE, J. T., y Holahan, C. K. «Psychological androgyny an sex role flexibility: a test of two hypothesis». Journal of Personality and Social Psychology, 1979, 37, 1631-1644.

Ho, R. «Sex, sex-role typing, and children's problem solving behavior». Journal of Social Psychology, 1981, 115, 219-226.

ICKES, W., y BARNES, R. D. «Boys and girls together and alienated: on enacting stereotyped sex roles in mixedsex dyads». Journal of Personality and Social Psychology, 1978, 36, 669-683.

KAGAN, J. "Acquisition and significance of sex tiping and sex-role identity», en M. L. Hoffman y L. W. Hoffman (eds.): Review of child development research (Vol. I). Nueva York: Russell Sage Foundation, 1964.

KELLY, J. A., y O’BrIEN, G. G., HoSFORD, R. «Sex-roles and social skills considerations for interpersonal adjustment». Psychology of Women Quarterly, 1981, 5 Suple., 748-766.

KLEIN, H., y WILLERMAN, L. «Psychological masculinity and femeninity and typical and maximal dominance expression in women». Journal of Personality and Social Psychology, 1979, 31, 2059-2070.

KOESTLER, A. The ghost in the machine. Londres: Hutchinson and Company, Ltd., 1967.

KOHLBERG, L. A. «A cognitive-developmental analysis of children's sex-role concepts and attitudes», en E. E. Maccoby (ed.): The development of sex differences. Stanford: Stanford University Press, 1966.

LAFRANCE, M., y CARMEN, B. «The nonverbal display of psychological androgyny». Journal of Perśonality and Social Psychology, 1980, 38, 36-49.

ORLOFSKY, J. L. «Psychological androgyny, sex-typing and sex-role ideology as predictors of male-female interpersonal attraction». Sex Roles, 1982, 8, 1057-1073.

ORLOFSKY, J. L., y WINDLE, M. T. «Sex-role orientation, behavioral adaptability and personal adjustment». Sex Roles, 1978, 4, 801-811.

Parsons, T., y BALES, R. F. Family, socialization and interaction process. Nueva York: Free Press of Glencoe, 1955.

SEBastín Herranz, J. La androginia como indice de flexibilidad comportamental. Tesis doctoral. Universidad Autónoma de Madrid, 1986.

SPENCE, J. T., y HelmReich, R. I. «On assessing "androgyny"». Sex Roles, 1979, 5, 721-737.

SPENCE, J. T.; Helmreich, R., y STAPP, J. «The Personal Attributes Questionnaire: a measure of sex role stereotypes and masculinity-femininity». ISAS Catalog of Selected Documents in Psychology, 1974, 4 (Ms. núm. 617).

SPENCE, J. T.; Helmreich, R., y StAPP, J. «Ratings of self and peers on sex role attributes and their relation to self-esteem and conceptions of masculinity and femeninity». Journal of Personality and Social Psychology, $1975,32,29-39$.

WIGGINGS, J. S., y Holzmuller, A. «Psychological androgyny and interpersonal behavior. Journal of Consulting and Clinical Psychology, 1978, 46, 40-52.

WigGins, J. S., y Holzmuller, A. «Further evidence on androgyny and interpersonal flexibility». Journal of Research in Personality, 1978, 15, 67-80. 